\title{
Berlin Declaration on Tuberculosis: High Level Follow-Up of High Priority Countries for TB Control in the WHO-EURO Region 'Double Trouble or Double Success? Bringing Together Diseases and Programs' - ein Symposiumsbericht
}

Autoren

Institute
S. Castell ${ }^{1}$, B. Hauer ${ }^{1,2}$, D. Manissero ${ }^{3}$, T. Ulrichs ${ }^{4}$, R. Zaleskis ${ }^{5}$, R. Loddenkemper ${ }^{1}$

Deutsches Zentralkomitee zur Bekämpfung der Tuberkulose, Berlin

Jetzige Adresse: Robert-Koch-Institut, Berlin

European Center for Disease Prevention and Control, Stockholm, Schweden

Koch-Metschnikow-Forum, Berlin

World Health Organization Regional Office for Europe, Kopenhagen, Dänemark

\section{Bibliografie}

DOI http://dx.doi.org/

10.1055/s-0029-1244160

Online-Publikation: 8. 6. 2010

Pneumologie 2010; 64:

422-429 @ Georg Thieme

Verlag KG Stuttgart · New York ISSN 0934-8387

\section{Korrespondenzadresse}

Prof. Dr. Dr. h.c.

\section{$R$. Loddenkemper}

DZK

Stralauer Platz 34

10243 Berlin

rloddenkemper@

dzk-tuberkulose.de

\section{Zusammenfassung}

\section{$\nabla$}

Die globale Tuberkulosesituation hat sich seit Anfang der 1990er-Jahre dramatisch verschlechtert. 2007 hat die WHO 18 Länder in der WHO-Europaregion als „high priority countries“ identifiziert und einen Plan vorgestellt, wie der Situation begegnet werden kann. Um weitere Lösungen zu befördern, fand 2007 ein Ministerforum der Staaten der WHO-Europaregion in Berlin statt, das in die gemeinsame Unterzeichnung der „Berliner Deklaration“ mündete. Im Oktober 2009 fand im Sinne dieser Deklaration unter Federführung des deutschen Bundesgesundheitsministeriums ein Symposium statt: „Berlin Declaration on Tuberculosis: High Level Follow-Up of High Priority Countries For TB Control In The WHO-EURO Region 'Double Trouble or Double Success? Bringing Together Diseases and Programs'“. Der vorliegende Beitrag fasst dieses zusammen. Neben aktuellen epidemiologischen Situationen der WHO-Europaregion mit zum Teil dramatischen Entwicklungen wurden psychosoziale Themen, die Rolle der EU und des „Global Fund to fight AIDS, Tuberculosis and Malaria“, die Bedeutung neuer Instrumente zur Tuberkulosebekämpfung und die Notwendigkeit weiteren politischen Engagements dargestellt.

\section{Einleitung}

$\nabla$

Die globale Tuberkulosesituation hat sich - nachdem noch Ende der 1970er-Jahre davon ausgegangen worden war, dass die Krankheit in Kürze besiegt sein würde - seit Anfang der 1990er-Jahre dramatisch verschlechtert. Hauptgründe für diese Entwicklung sind die HIV-Epidemie, das zunehmende Auftreten medikamentenresistenter Tuberkulose-Bakterienstämme (MDR-, XDR- und mittlerweile sogar XXDR-Tuberkulose ${ }^{1}$ ) sowie die politischen Veränderungen in den Staaten der ehemaligen Sowjetunion mit den damit einhergehenden Auswirkungen auf die Gesellschaft, die

\section{Abstract \\ $\nabla$}

The global tuberculosis (TB) situation has deteriorated dramatically since the beginning of the 1990s. In 2007, the WHO identified 18 countries of the WHO European Region as 'high priority countries' and introduced a plan for these countries to improve the situation. To further promote solutions a WHO European Ministerial Forum 'All against Tuberculosis' took place in Berlin in 2007 and resulted in the 'Berlin Declaration' which was commonly endorsed. In October 2009 a meeting was organized by the German Ministry of Health under the title „Berlin Declaration on Tuberculosis: High Level Follow-Up of High Priority Countries for TB Control in the WHO-EURO Region 'Double Trouble or Double Success? Bringing together Diseases and Programs'“. This article summarizes the symposium. Besides reporting on the recent epidemiological situation of the WHO-EURO Region (with partly dramatically developments) presentations on psychosocial issues, the role of the EU and the 'Global Fund to Fight AIDS, Tuberculosis and Malaria', the importance of new tools for the fight against tuberculosis and the need for further political commitment were given.

medizinischen Versorgungsstrukturen und Kontrollfunktionen des Öffentlichen Gesundheitsdienstes.

Sehr hohe Tuberkuloseinzidenzen finden sich in den südlich der Sahara gelegenen afrikanischen sowie vielen südostasiatischen Ländern. Dies ist

\footnotetext{
${ }^{1}$ MDR (multidrug-resistant): Resistenz mindestens gegenüber Isoniazid und Rifampicin, XDR (extensively drug-resistant): MDR und zusätzlich Resistenz gegenüber einem Fluorchinolon und wenigstens gegen eines der injizierbaren Zweitrangmedikamente Capreomycin, Kanamycin und Amikacin, XXDR (extremely drug-resistant): Resistenz gegenüber allen Erst- und Zweitrangmedikamenten.
} 


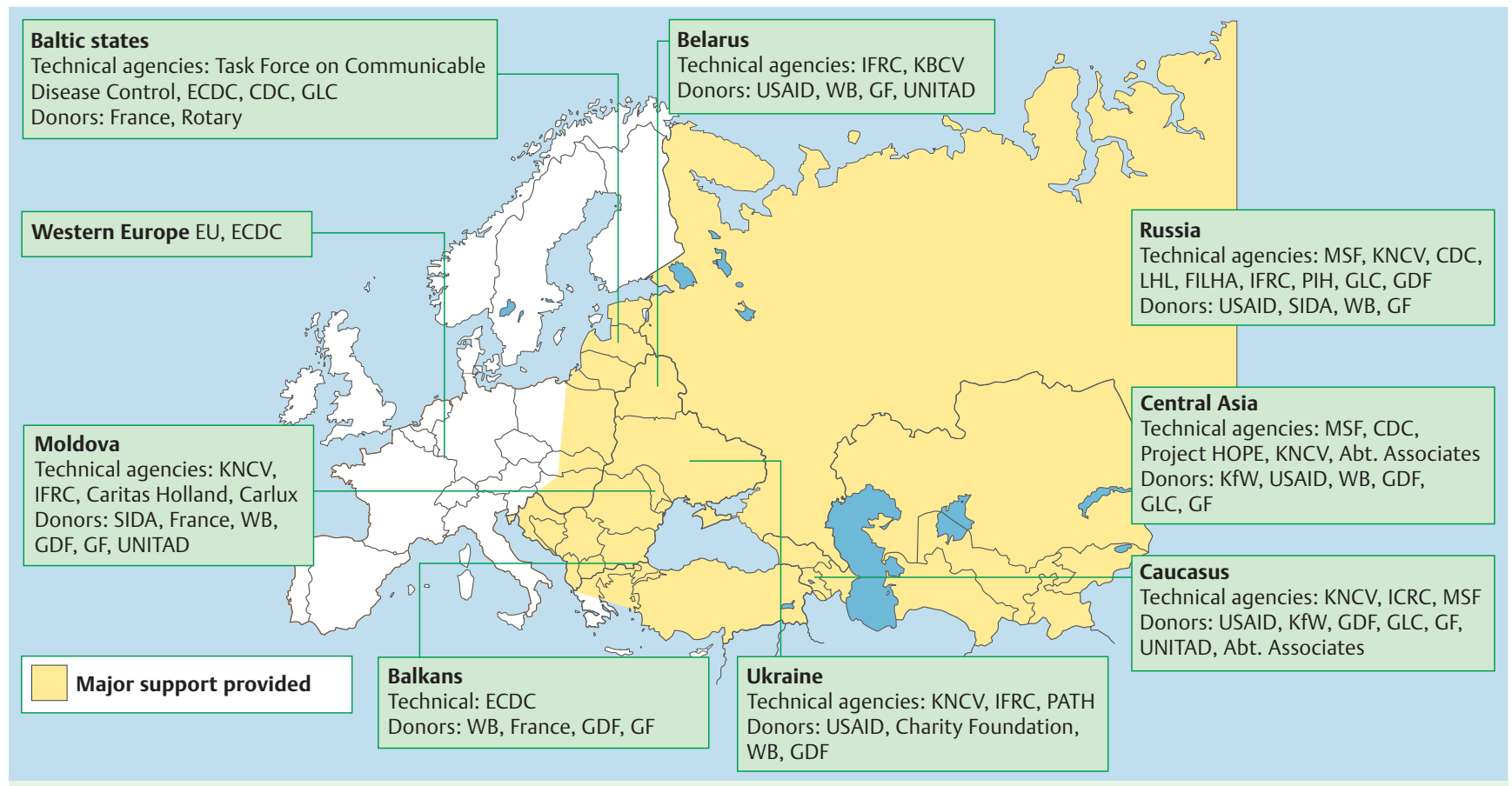

Abb. 1 Partner der Tuberkulosekontrolle in der WHO-Europaregion (nach Zaleskis).

vor allem durch die hohen HIV-Infektionsraten verursacht, da HIV-Infizierte besonders empfänglich für die Entwicklung von Tuberkulose sind. Daneben rückte zunehmend die Situation in der nach der Weltgesundheitsorganisation (WHO) definierten Europaregion (bestehend aus 53 Ländern $^{2}$ ) in den Fokus der Aufmerksamkeit, da hier die Länder mit den weltweit höchsten MDR-TB-Raten vertreten sind. Dies hat zu einem Ausbau der Kooperationen von sowohl staatlichen als auch nicht-staatlichen Institutionen und Organisationen geführt, die in der Region in allen Bereichen der Tuberkulosebekämpfung aktiv sind ( $\bullet$ Abb. 1). 2007 hat die WHO 18 Länder in dieser Region als „high priority countries“" identifiziert und einen Plan vorgestellt, wie regionsspezifisch bis 2015 der Situation begegnet werden kann [1]. Die Europäische Union (EU) wird durch die Osterweiterung und die Nachbarschaft zu den betroffenen Ländern unmittelbar mit der Problematik konfrontiert. Aus diesem Grund wurde das European Centre for Disease Prevention and Control (ECDC) vom EUKommissar für Gesundheit beauftragt, federführend einen Aktionsplan zu erarbeiten, der im Februar 2008 veröffentlicht wurde [2]. In Anerkennung der Problematik und der dringlichen Notwendigkeit einer konzertierten Lösungsstrategie fand im Oktober 2007 unter Gastgeberschaft des deutschen Bundesgesundheitsministerium das europäische WHO-Ministerforum „Alle gegen Tuberkulose“ mit Teilnahme von 49 der 53 Gesundheitsminister der Staaten der WHO-Europaregion in Berlin statt. Dieses mündete in der gemeinsamen Unterzeichnung der „Berliner Deklaration“, in welcher die vielschichtigen Probleme benannt, der Handlungs- und Forschungsbedarf klar umrissen sowie die Absicht aller Länder formuliert ist, die Situation unter Berücksichtigung der Stopp-TB-Strategie zu verbessern $[3,4]$. Teil der

\footnotetext{
${ }^{2}$ Im Folgenden als WHO-Europaregion bezeichnet.

${ }^{3} \mathrm{Zu}$ den 18 Hoch-Prioritäts-Ländern zählen: Armenien, Aserbaidschan, Weißrussland, Bulgarien, Estland, Georgien, Kasachstan, Kirgisische Republik, Lettland, Litauen, Moldawien, Rumänien, Russische Föderation, Tadschikistan, Türkei, Turkmenistan, Ukraine and Usbekistan.
}

Deklaration war die Forderung, ab 2009 die Fortschritte der Region zweijährlich zu bewerten. Um diesen Evaluationsprozess zu unterstützen, fand im Vorfeld des ersten World Health Summit am 15. Oktober 2009 in Berlin unter Federführung des deutschen Bundesgesundheitsministeriums ein Symposium statt. Der Titel lautete: „Berlin Declaration on Tuberculosis: High Level FollowUp of High Priority Countries For TB Control In The WHO-EURO Region 'Double Trouble or Double Success? Bringing Together Diseases and Programs'“. Der vorliegende Beitrag fasst die Inhalte und wesentlichen Ergebnisse des Symposiums zusammen.

\section{Aktuelle epidemiologische Situation in der WHO-Europaregion}

2007 lag die geschätzte Tuberkuloseinzidenz in der WHO-Europaregion bei 49 Neuerkrankungen/100000 Einwohner. Für die Gesamtregion zeigte sich in den vergangenen Jahren eine Stabilisierung, dies trifft jedoch nicht auf Osteuropa zu ( $\bullet$ Abb. 2). Beispielsweise stieg in der Ukraine die Anzahl der Neuerkrankungen pro Jahr und 100000 Einwohner von 41 (1990) auf $102 \mathrm{im} \mathrm{Jahr}$ 2007 [5].

In $\odot$ Abb. 3 wird die Dimension der Problematik anhand der Absolutzahlen deutlich. Eine Tuberkulosebehandlung gemäß der DOTS-Strategie wird in der WHO-Europaregion schätzungsweise bei $75 \%$ der Therapien durchgeführt, der Behandlungserfolg liegt bei $70 \%$ und befindet sich damit unter dem angestrebten Ziel der WHO von 85\% [5].

Der Anteil der 2007 an Tuberkulose neu erkrankten Personen, die zusätzlich mit dem HI-Virus infiziert waren, wird auf 9,8\% geschätzt, wobei sich hier ebenfalls regional große Unterschiede zeigen (siehe $\bullet$ Abb. 4). In der Russischen Föderation liegt dieser Anteil bei $16 \%$, in Estland bei 17\%, in Portugal und der Ukraine bei $20 \%$, während in Deutschland oder Großbritannien dieser Anteil wesentlich niedriger geschätzt wird (2,3\% bzw. 3,3\%) [5]. 


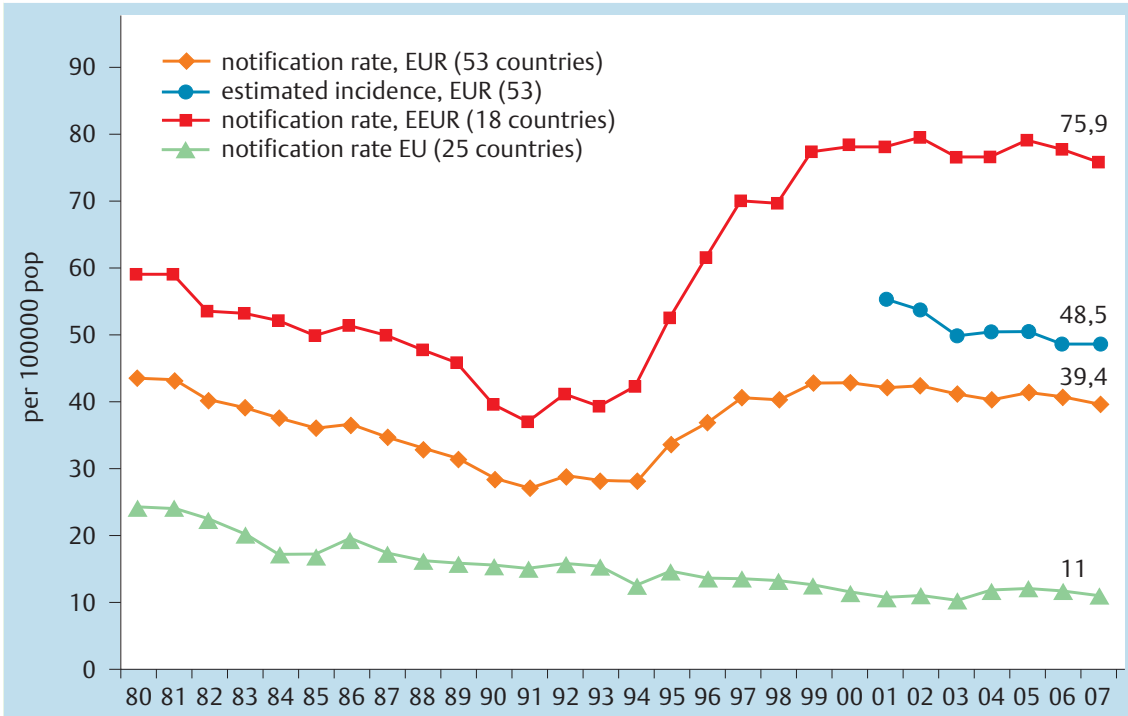

Abb. 2 Tuberkulose in der WHO-Europaregion, 1980-2007 (nach Zaleskis).

80818283848586878889909192939495969798990001020304050607

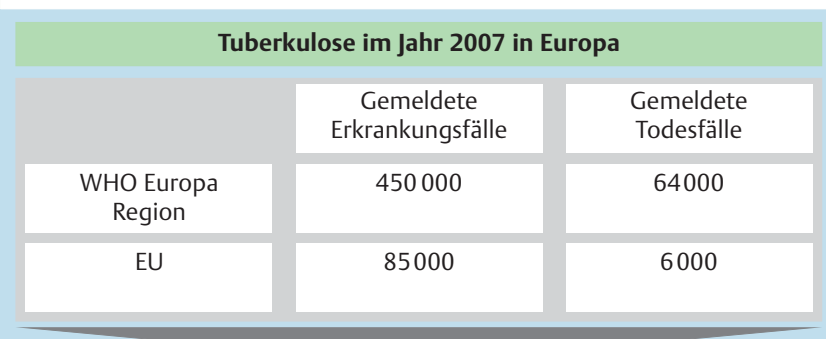

jede Stunde 50 neue Fälle, 7 Tote

Therapieerfolgsrate: $70 \%$

Abb. 3 Epidemiologische Eckdaten zur Tuberkulosesituation in Europa, modifiziert nach Ryan.
15 und damit mehr als die Hälfte der gemäß WHO weltweit 27 MDR-TB-,high burden“-Länder liegen in den osteuropäischen Ländern der WHO-Europaregion [5,6] (siehe $\bullet$ Abb.5). Der Nachweis von multiresistenten Stämmen wurde für die gesamte Region bei nicht vorbehandelten Patienten bei $10 \%$ geführt, bei Patienten mit antituberkulotischer Vorbehandlung bei 43\% [5]. In vielen der am meisten betroffenen Ländern ist dabei die MDR-TB häufig mit der HIV-Infektion verknüpft (Beispiele siehe - Tab. 1).

Die Schätzungen der WHO zu den Resistenzraten beruhen dabei in den Ländern ohne flächendeckende kontinuierliche Erhebungen auf regionalen Surveys bzw. Datenerfassungen im Rahmen von Labornetzwerken. Die korrekte Einschätzung der Resistenzraten würde die verlässliche Resistenztestung aller kulturell positiven Patienten in qualitätskontrollierten Laboratorien erfordern. Diese Voraussetzungen sind in vielen Staaten der WHO-Europaregion jedoch nicht gegeben.

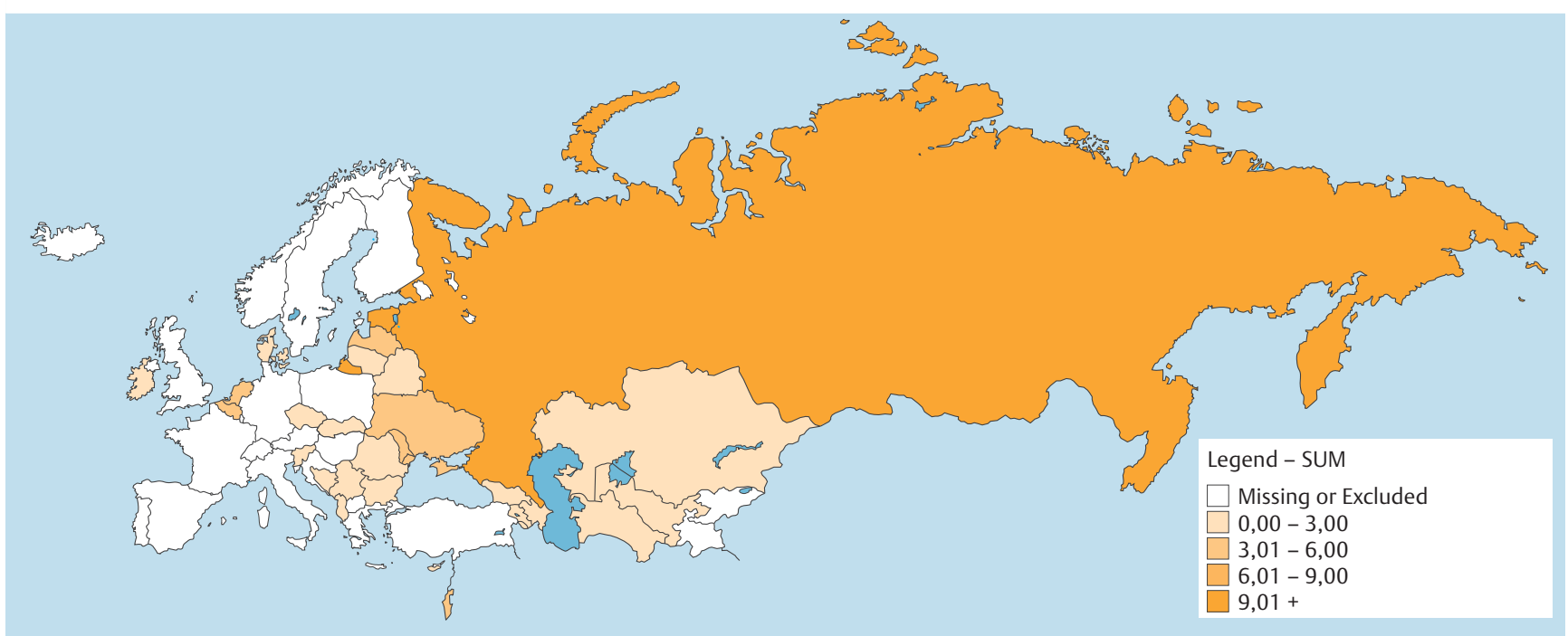

Abb. 4 HIV-Prävalenz bei TB-Erkrankten in der WHO-Europaregion 2007 (nach Zaleskis). 


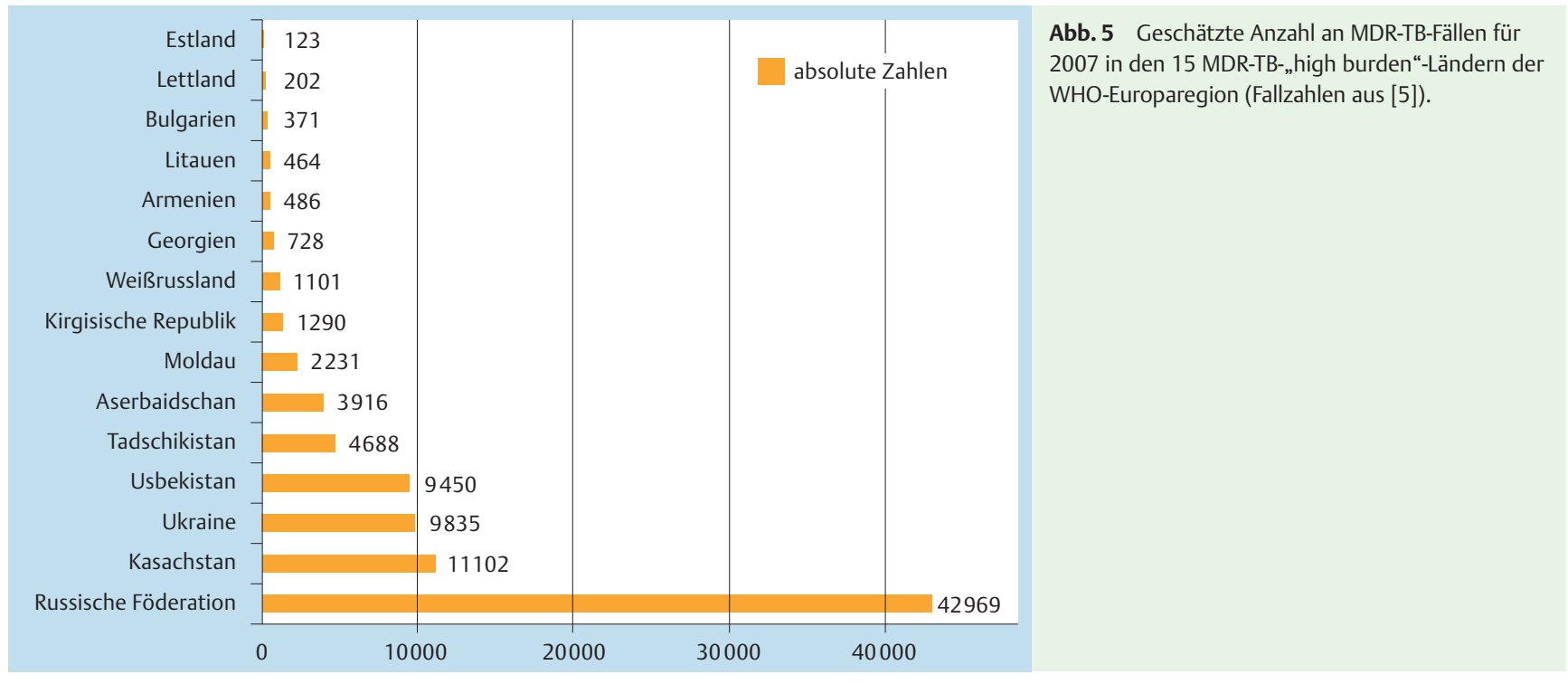

\begin{tabular}{|c|c|c|c|c|c|}
\hline & \multirow{2}{*}{$\begin{array}{l}\text { Getestete TB Fälle } \\
\text { n }\end{array}$} & \multicolumn{2}{|c|}{ Jegliche Resistenz } & \multicolumn{2}{|c|}{ MDR-TB } \\
\hline & & $\mathbf{n}$ & $\%$ & n & $\%$ \\
\hline \multicolumn{6}{|l|}{ Lettland; $2001-2005$} \\
\hline HIV-Status unbekannt & 5162 & 1782 & 34,5 & 765 & 14,8 \\
\hline HIV-positiv & 148 & 66 & 44,6 & 39 & 26,4 \\
\hline OR & & \multicolumn{2}{|c|}{1,5} & \multicolumn{2}{|c|}{2,1} \\
\hline p-Wert (95\%-KI der OR) & & $<0,05$ & $2,1)$ & $<0,0$ & $-3,0)$ \\
\hline \multicolumn{6}{|l|}{ Donetsk (Ukraine); 2006} \\
\hline HIV-negativ & 1143 & 551 & 48,2 & 272 & 23,8 \\
\hline HIV-positiv & 307 & 173 & 56,4 & 97 & 31,6 \\
\hline OR & & \multicolumn{2}{|c|}{1,4} & \multicolumn{2}{|c|}{1,5} \\
\hline p-Wert (95\%-KI der OR) & & $<0,05$ & $1,8)$ & $<0,0$ & $-2,0)$ \\
\hline
\end{tabular}

Wenn die Daten der einzelnen Länder der Region betrachtet werden, zeigt sich eine heterogene Situation: So zählt z.B. Deutschland, wie die meisten westeuropäischen Nationen, zu den Niedrig-Inzidenzländern ${ }^{4}$ [7] (Inzidenz 2007:6/100000; davon geschätzt HIV-infiziert: 2,3\%; MDR-Anteil 1,8\% bei Erstbehandelten, $12 \%$ bei vorbehandelten Patienten). $\mathrm{Zu}$ den Ländern mit einer hohen Tuberkulose-Erkrankungsrate, aber niedrigen Resistenz- und HIV-Koinfektionsraten zählt unter anderen Rumänien mit einer TB-Inzidenz 2007 von 115/100000 (davon HIV-infiziert: 2,4\%; MDR-Anteil 2,8\% bei Erstbehandelten, $11 \%$ bei vorbehandelten Patienten). Ein Land mit hohen Inzidenzen und einem hohen Anteil an Resistenz- und TB/HIV-Koinfektionsraten ist z.B. die Ukraine (Inzidenz 2007:102/100000, davon HIV-infiziert: 20\%; MDR-Anteil 16\% bei Erstbehandelten, $44 \%$ bei vorbehandelten Patienten) $)^{5}$.

Die geschätzte Tuberkulosemortalität lag 2007 - um bei den Beispielen zu bleiben - in Deutschland bei < 1/100000, in Rumänien bei 16/100000 und in der Ukraine bei 15/100000 [5]. Auch hier zeigt sich also eine deutlich heterogene Situation. Die Ein-Jahres-Mortalität für TB/HIV-Koinfizierte wird für Osteuropa auf $27 \%$ und für Nord- bzw. Südeuropa auf $7 \%$ bzw. 9\% geschätzt [8]. Der Wert der epidemiologischen Daten zu den TB/HIV-Koinfektionsraten wird dadurch eingeschränkt, dass HIV-Testungen bei

\footnotetext{
${ }^{4}$ Länder mit einer per definitionem niedrigen Inzidenz haben weniger als 20 TB-Neuerkrankungen/100000 Einwohner und Jahr, mit einer mittleren Inzidenz 20-100 Neuerkrankungen/100000 und mit einer hohen Inzidenz mehr als 100/100000.

${ }^{5}$ Alle Zahlen sind Schätzwerte der WHO aus [5.]
}

Tuberkulosekranken noch immer zu selten angeboten bzw. durchgeführt werden (geschätzter Ist-Zustand $<10 \%$, angestrebt $>80 \%$, Paul Sommerfeld von TB Alert). Zudem sind aus Datenschutzgründen HIV- und TB-Melderegister in vielen Ländern nicht miteinander verknüpfbar, was eine umfassende und gesamteuropäische Auswertung erschwert (Davide Manissero, TBCoordinator, ECDC). So liegt der bereits erwähnte geschätzte Anteil der Koinfektionen bei TB-Neuerkrankten bei 9,8\% (absolut: 42322 Fälle), tatsächlich gemeldet wurden hiervon jedoch nur 6710 Fälle. Folglich werden nur 16\% der vermuteten Koinfektionen gemeldet (John-F. Ryan, Head of Health Threats Unit, European Commission, Generaldirektion Gesundheit und Verbraucher). Vorbehaltlich der schwierigen Datenerhebung zeigen einige Länder der WHO-Europaregion einen besorgniserregenden Anstieg der HIV-Infektionsrate, sowohl in der Gesamtbevölkerung ( $\bullet$ Abb. 6 zeigt den Trend für die WHO-Europaregion) als auch bei TB-Erkrankten. So stieg die HIV/TB-Koinfektionsrate in Lettland von 1,3\% im Jahr 2001 auf 4,4\% im Jahr 2007. Im Gegensatz zu den meisten anderen westeuropäischen Nationen zeigen sich in Portugal für denselben Zeitraum außerordentlich hohe Koinfektionsraten von bis zu $15,5 \%$.

Bei der Bewertung dieser aktuellen epidemiologischen Datenlage der WHO-Europaregion weist Marc Danzon (Regional Director, WHO Regional Office for Europe) darauf hin, dass sich aufgrund der gegenwärtigen ökonomischen und sozialen Krise der positive Trend der vergangenen Jahre, i.S. einer Stabilisierung der Tuberkulose-Inzidenzen und -Prävalenzen, nicht wird halten lassen, 


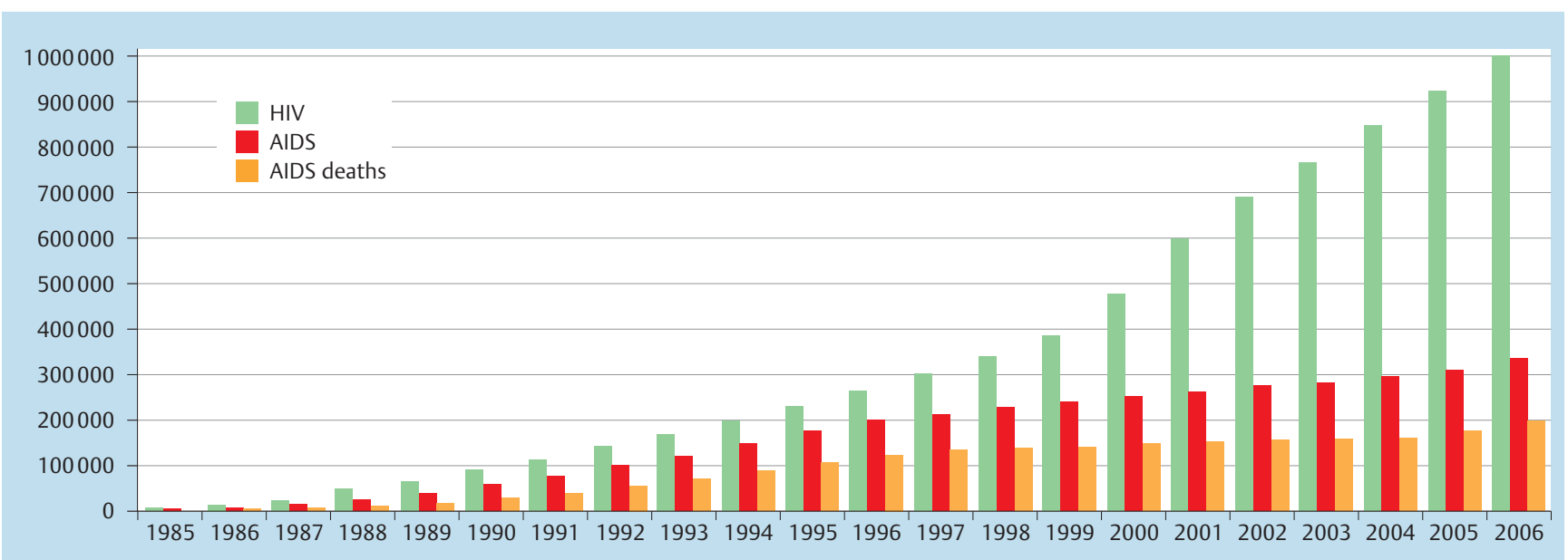

Abb. 6 Kumulative Anzahl aller in der WHO-Europaregion gemeldeten HIV-, AIDS- und AIDS-Todesfälle (nach Zaleskis).

sodass gegenwärtig von einer „unstable stabilization“ auszugehen ist.

Insgesamt wird die TB-Situation in der WHO-Europaregion weiterhin als bedrohlich eingeschätzt, besonders gilt dies für Osteuropa. Hinsichtlich der HIV-Situation ist von einer Epidemie auszugehen, ein weiterer Anstieg der Infektionsraten ist zu erwarten. Darüber hinaus erhalten in der WHO-Europaregion nur schätzungsweise $2 \%$ der mit TB und HIV doppelt infizierten Patienten eine antiretrovirale Therapie (Zaleskis, Regional Adviser, TB Control, WHO Regional Office for Europe).

Für weite Teile der WHO-Europaregion werden somit die Millennium Development Goals wahrscheinlich nicht erreicht werden. Diese beinhalten die Halbierung der Tuberkuloseprävalenz und Mortalität im Vergleich zu 1990 bis 2015, den flächendeckenden und freien Zugang zur HIV-Therapie bis 2010, die Stabilisierung bzw. Trendwende sowohl hinsichtlich der Tuberkuloseinzidenzen als auch der Verbreitung von HIV bis 2015. Dies ist ein aus Sicht aller Teilnehmer völlig unakzeptabler Zustand, der dringend verbessert werden muss.

\section{Themenschwerpunkte}

Die damals amtierende deutsche Gesundheitsministerin, Ulla Schmidt, betonte in ihrer Begrüßungsansprache die globale Bedrohung durch die Tuberkulose und insbesondere auch durch die HIV/TB-Koinfektion. Als wesentliche Gründe nannte sie psychosoziale Ursachen, mangelnde Infrastruktur und Ressourcen sowie Ausgrenzung und Stigmatisierung. Deutschland engagiert sich vor diesem Hintergrund international in Bereichen wie der Verbesserung der Diagnostik, der Gesundheitssystemstärkung und der HIV-Aufklärung. So ist Deutschland beispielsweise über Kooperations- und Entwicklungsprojekte in afrikanischen Ländern aktiv, fördert, unter Beteiligung von Nichtregierungsorganisationen, bilaterale Projekte und hat 2008 und 2009 den Globalen Fonds gegen AIDS, Tuberkulose und Malaria (Global Fund) mit jeweils 200 Millionen Euro unterstützt. ${ }^{6}$

\footnotetext{
${ }^{6}$ Mündliche Mitteilung Jochen Böhmer, Bundesministerium für wirtschaftliche Entwicklung und Zusammenarbeit.
}

\section{Tuberkulose allgemein}

Die in Zeiten der globalen Krise mehr denn je notwendige Stärkung der Gesundheitssysteme, insbesondere auch der primären medizinischen Versorgungsstrukturen, die herausragende Bedeutung gut ausgebildeten medizinischen Personals und der grundsätzlich erforderliche politische Wille, wurden von den verschiedenen Rednern ebenso thematisiert wie die - lange Zeit unterschätzte und vernachlässigte - Bedeutung der Zivilgesellschaft (Danzon). Die Berliner Deklaration wird in diesem Kontext als gute Arbeitsgrundlage angesehen, die weiter implementiert werden muss.

Die anhaltende Problematik der MDR-TB macht deutlich (Danzon), dass es noch grundsätzlichen Handlungsbedarf gibt und die (europäischen) Gesundheits- und Sozialsysteme zum Teil versagen. Hier ist die Solidarität reicher Länder gefragt, um der globalen Gefährdung durch einzelne schwache Systeme wirkungsvoll und nachhaltig zu begegnen. Dabei zeigt gerade die unheilvolle Verbindung von MDR-TB mit der HIV-Infektion, dass die Lösungsansätze zur Bekämpfung von Tuberkulose und HIV verbunden werden müssen. Dies ist ein Aspekt, der noch zu wenig berücksichtigt wird.

\section{Das Problem der Stigmatisierung}

Für TB-Patienten und mehr noch für HIV-Infizierte spielt das Problem der Stigmatisierung eine wichtige Rolle. Paul Sommerfeld bemerkte dazu einleitend „Many people talk about stigma, but don't tell us what you can do about it" (Viele Leute sprechen über Stigma, aber sagen uns nicht, was man dagegen tun kann.), und dass das komplexe Thema „Stigmatisierung“ über die Zeit, zwischen Kulturen und Regionen bis hin zu Individuen variiert, sodass hier sehr spezifische Lösungen gefunden werden müssen. Erfolgreich sind insbesondere Peer-to-peer-Ansätze: (Ehemalige) TB/HIV-koinfizierte Patienten teilen persönliche Erfahrung und Wissen („Our TB has been cured even if we are HIV positive“, Unsere TB wurde erfolgreich behandelt, obwohl wir HIV-positiv sind.). Die Bereitschaft, getestet und behandelt zu werden, wird auf diese Weise gefördert. Dies erfordert die Kooperation mit lokalen, Gemeinde-nahen Organisationen.

\section{Die Bedeutung vulnerabler Gruppen}

Als Beispiel für eine vulnerable und schwer erreichbare Gruppe setzte sich Peter Gondrie (Executive Director KNCV Niederlande) mit der Situation von Gefangenen auseinander, deren stärkere 
Berücksichtigung ebenfalls in der Berliner Deklaration festgeschrieben ist. In vielen Ländern hat sich die Situation in den vergangenen Jahren durch Initiativen wie das Health in Prison Project (HIPP) verbessern lassen, ist jedoch immer noch hochproblematisch.

Darüber hinaus wurde das Thema Migration angesprochen. In vielen Ländern Europas sind mittlerweile eine Mehrzahl (z.B. Schweden 78\%) bzw. ein Großteil (z. B. Deutschland 42\%) der tuberkulosekranken Menschen im Ausland geboren. EU-weit liegt dieser Anteil bei 24\% (inkl. unbekannter Herkunft) [7]. Dies bedeutet, dass den Tuberkulose-gefährdeten Immigranten besondere Aufmerksamkeit gewidmet werden muss.

\section{Die Rolle der EU}

Was die Situation der Migranten angeht, kann die Herangehensweise dabei aufgrund der europäischen Reisefreiheit, die durch das Schengener Abkommen besteht, nur gesamteuropäisch sein (Ryan). Im Rahmen der Neuformierung der Europäischen Kommission wurden Prioritäten für die nächsten fünf Jahre festgelegt, wobei hier die Diskussion innerhalb des Symposiums als ein guter Ausgangspunkt anzusehen ist, die Themen HIV/TB angemessen zu berücksichtigen. Das aktive Engagement von Seiten der EU ist zur erfolgreichen Umsetzung der Berliner Deklaration von großer Bedeutung.

\section{Die Rolle des Global Fund}

Der „Global Fund to fight AIDS, Tuberculosis and Malaria“ (GF) stellt zwei Drittel der weltweiten Finanzierung des Kampfes gegen TB (www.theglobalfund.org/en/about, 22.10. 09). Rifat Atun (GF) wies in seinem Vortrag darauf hin, dass die finanzielle Unterstützung zukünftig für manche Länder der WHO-Europaregion reduziert oder sogar eingestellt werden wird, da die geforderten Kriterien aufgrund der sozioökonomischen Entwicklung der Länder nicht mehr erfüllt werden (Beispiel Rumänien mit einer Tuberkuloseinzidenz 2007 von 115/100000). Die Finanzierung von TB- und HIV-Programmen durch den Global Fund ist primär von sozioökonomischen Faktoren der Empfängerländer abhängig und weniger von der epidemiologischen Situation. Die Auswirkungen stellen eine große Herausforderung an die EU dar. Offensichtlich scheint manchen Ländern auch die Erfüllung der Anforderungen bei der Antragstellung Probleme zu bereiten.

\section{Probleme bei der Implementierung neuer Instrumente zur Tuberkulose-Bekämpfung}

Christy Hanson von der „STOP-TB Re-Tooling Task Force“ (stoptbretooling@who.int) berichtete, dass viele Länder mit der Implementierung neuer Instrumente oder Strategien zur Tuberkulose-Kontrolle warten, bis diese von der WHO offiziell empfohlen werden. Auf diese Weise würden unnötige und potenziell folgenschwere Verzögerungen entstehen. Unter Umständen seien vor der Einführung neuer Richtlinien in den einzelnen Ländern zur Prävention, Diagnostik oder Therapie spezifische Anpassungen notwendig. Hanson betonte die erhebliche Bedeutung des Zugangs zu entsprechenden neuen Technologien für alle Betroffenen. Verbesserungsbedarf besteht auch bezüglich der Standardisierung der Evaluation von neuen Diagnostika. Ein entsprechender Entwurf wurde - im Sinne eines international einheitlichen Vorgehens - von der „New Diagnostic Working Group“ der „Stop TB Partnership“ erstellt.

\section{Aktuelle Entwicklungen am Beispiel des}

„high priority“" Landes Ukraine

Als Beispiel für ein „High Priority“-Land wurde die Situation der Ukraine präsentiert, welche die höchsten HIV-Infektionsraten in Europa aufweist [10] (HIV-Prävalenz der 15-49-Jährigen 2007: $1,6 \%$ [11], zum Vergleich Deutschland 0,1\%). Bis zu 21\% der an Tuberkulose Erkrankten sind zusätzlich mit HIV infiziert [5], dabei ist die Mortalität unter den koinfizierten Patienten hoch. Das Tuberkulose-Management weist nach Aussage von Kateryna Gamazina (Country Director PATH Ukraine) deutliche Defizite auf: Die Behandlung nach DOTS-Kriterien wird nur in 29\% des Landes umgesetzt, außerdem gibt es keine zuverlässigen Daten zum Therapieerfolg [12]. Die MDR-TB-Raten gehören zu den höchsten in Europa.

Die dringend notwendige Zusammenarbeit zwischen TB- und HIV-Programmen war bisher kaum verwirklicht worden. Mittels eines Projektes, das von PATH („Program for Appropriate Technology in Health“), einer internationalen Nonprofit-Organisation, eingeführt wurde, konnte jedoch über Jahre eine teilweise enge Kooperation entwickelt werden. Dabei wurde im Besonderen das Misstrauen der betroffenen Bevölkerung gegenüber den Mitarbeitern des Gesundheitssystems sowie die Diskriminierung von tuberkulosekranken Menschen berücksichtigt. Diese liegt in der Assoziation der Erkrankung mit Armut, Obdachlosigkeit, Drogengebrauch, Alkoholkrankheit und Gefängnisvergangenheit begründet. Viele Patienten fürchten den Verlust des Arbeitsplatzes, wenn sie offiziell mit Tuberkulose diagnostiziert werden. Außerdem ist die Meinung verbreitet, dass Tuberkulose nicht geheilt werden kann oder dass die Behandlung zu teuer ist.

Auf der Basis dieser Erkenntnisse wurden spezifische Kommunikationsstrategien entwickelt. Das Programm beinhaltet ein gezieltes Training von Ärzten, das einerseits aktuelle klinische Informationen und andererseits eine Verbesserung der Kommunikationsfähigkeit und Kenntnisse in psychologischer Beratung vermittelt, sodass Patienten - gerade aus Problemgruppen - besser unterstützt werden können (www.path.org). Außerdem gibt es Informationsmaterial für Patienten, ihre Familien und die allgemeine Öffentlichkeit. Diese Herangehensweise, bei der verschiedene Gruppen angesprochen und mehrere Ebenen eingebunden werden, hat sich als sehr erfolgreich erwiesen. So konnten z.B. die Therapieadhärenz verbessert sowie die Therapieabbruchraten deutlich gesenkt werden, was langfristig zur Abnahme der Resistenzraten führen wird. Die Forderung der Berliner Deklaration „Focused action is needed to tackle MDR/XDR-TB and TB/HIV co-infection“ kann mit Hilfe solcher Programme erreicht werden. Grundvoraussetzung ist die ausreichende finanzielle Unterstützung, da Nichtregierungsorganisationen von externer Finanzierung abhängig sind.

\section{Round Table Discussion}

$\nabla$

Abgeschlossen wurde die Veranstaltung mit einer „round table discussion“, wobei die teilnehmenden Gesundheitsminister und -ministerinnen bzw. deren Vertreter ein kurzes Statement zur aktuellen Situation und zu den Fortschritten der letzten Jahre abgaben. Vertreten waren Bulgarien, die Ukraine, die Russische Föderation, Weißrussland, die Türkei, Moldawien, Kasachstan, Rumänien sowie das Gastgeberland Deutschland.

Alle Ländervertreter sehen in der Bekämpfung von HIV/TB eine Priorität. Sie bekennen sich zu den Inhalten der Berliner Deklaration und bemühen sich um deren Implementierung und die Wei- 
terentwicklung von nationalen TB- und HIV-Kontrollprogrammen. Dabei wurde die verstärkte Berücksichtigung Tuberkulose-/HIV-gefährdeter Risikogruppen deutlich. Größtenteils wird dieser Prozess von nationalen Koordinierungsgremien, unter Einbindung von Nichtregierungsorganisationen und Vertretern der Zivilbevölkerung, unterstützt. Verschiedene nationale Projekte und Aktivitäten wurden seit Unterzeichnung der Berliner Deklaration initiiert, wobei die Ressourcenknappheit, welche sich aufgrund der globalen wirtschaftlichen Situation noch weiter verschärft hat, ein Problem darstellt. Es wird an die EU bzw. internationale Institutionen - insbesondere an den Global Fund - appelliert, die finanzielle Unterstützung aufrechtzuerhalten, da die rein nationale Finanzierung nicht ausreichend ist.

Für Deutschland betonte Theo Schröder, Staatssekretär im Bundesministerium für Gesundheit, dass ressourcenstarke Länder wie Deutschland über die Landesgrenzen hinaus aktiv sein müssen. Grundsätzlich müssen integrative Strategien verfolgt werden, um überall eine Verzahnung von spezifischen Programmen mit dem allgemeinen Gesundheitswesen zu erreichen. Die Überführung begrenzt finanzierter Projekte in nachhaltige Maßnahmen müsse gewährleistet sein, um langfristig ein flächendeckendes und leistungsfähiges System zur Bekämpfung von HIV/TB aufzubauen.

\section{Schlussfolgerungen und Aussichten}

$\nabla$

Tuberkulose und HIV stellen in der WHO-Europaregion weiterhin eine ernst zu nehmende Bedrohung dar. Die Aussagen und Handlungsabsichten der Berliner Deklaration von 2007 werden deswegen von allen Teilnehmern des Forums nochmals unterstrichen und sollen weiterhin prioritär behandelt und implementiert werden.

Folgende Aspekte wurden besonders hervorgehoben:

- Nur mittels nationaler und länderübergreifender Kooperationen, Forschungsanstrengungen, Wissenstransfer und ausreichender finanzieller Ausstattung können die angestrebten Ziele erreicht werden. Das Engagement der Europäischen Kommission und des Global Funds wird begrüßt und ist unverzichtbar.

- In den vergangenen zwei Jahren sind die Länder der Region in Anerkennung der Tuberkulose- und HIV-Problematik auf verschiedenen Ebenen aktiv geworden, so beispielsweise im Rahmen der Implementierung nationaler TB-Kontrollprogramme, über die Bildung koordinierender Komitees (unter Einbindung verschiedener, auch nicht-staatlicher Organisationen), in der systematischen Anwendung von krankheitsund subgruppenspezifischen Programmen oder in der verstärkten Ausbildung von im Gesundheitswesen Tätiger. Das Potenzial eines konstruktiven Zusammenwirkens von Kooperationspartnern aus unterschiedlichen Bereichen wurde vielerorts erkannt und genutzt, um die Situation von Tuberkulose- und HIV-Patienten zu verbessern. Dieser Ansatz bedarf noch der Umsetzung in größerem Rahmen. Solche integrierenden Ansätze kombinieren die vertikale Fokussierung auf TB-relevante Anliegen mit der horizontalen Stärkung der Gesundheitssysteme und lassen zugleich den einzelnen Menschen in seinem Umfeld nicht außer Betracht [13].

- Im Rahmen des Symposiums wurde der Diskussionsbedarf bezüglich der Förderkriterien des Global Funds deutlich. Es wurde hinterfragt, ob die Gewichtung einer schwierigen epi- demiologischen Situation eines Landes ausreichend berücksichtigt ist.

- Den psychosozialen Faktoren wird zunehmend Aufmerksamkeit geschenkt. Die Verminderung der Stigmatisierung von Menschen mit HIV/AIDS und/oder TB hat sich als Schlüsselkomponente erwiesen, da sich so bei den Betroffenen die Bereitschaft erhöht, sich testen und effektiv behandeln zu lassen.

- Als gesonderter Aspekt wurde die Bedeutung der HIV-Testung von Tuberkulosepatienten herausgestellt, da nur so die Betroffenen optimale medizinische Betreuung erhalten können und sich die Mortalität senken lässt. Die Kenntnis des eigenen HIV-positiven Status kann weitere Infektionen verhindern helfen, daneben sind primärpräventive Maßnahmen und ausreichende Aufklärung als Schlüsselelemente einer erfolgreichen HIV-Bekämpfung essenziell. Außerdem ist die Optimierung bzw. der Aufbau einer HIV-Surveillance notwendig, um eine bessere Einschätzung der Krankheitslast, der erforderlichen Maßnahmen und des notwendigen Finanzbedarfs zu erreichen.

- Ein politisches Engagement, das eine wirksame TB- und HIV-Bekämpfung erreichen will, muss über Ländergrenzen hinausgehen, und darf sich nicht in rein medizinischen Strategien erschöpfen, sondern muss soziale und psychologische Faktoren wesentlich mit in Betracht ziehen. Dies bedeutet unter anderem die Zusammenarbeit mit Nichtregierungsorganisationen, zivilgesellschaftlichen Gruppen und Patientenvertretern ebenso wie ein verstärktes Engagement bei den Themen soziale Benachteiligung, Geschlechtergleichstellung, Situation von Migranten, Wohnsituation, Zugang zu sozialen und Gesundheitsdiensten und Verminderung von Stigmatisierung.

- Nur durch die zügige Implementierung neuer Instrumente der Tuberkulose- und HIV-Bekämpfung und den ungehinderten Zugang dazu wird die Situation zu beherrschen sein.

(Für einen aktuellen Überblick über den Stand der medizinischen Entwicklung siehe [14]).

Zusammenfassend hat die Veranstaltung in Berlin gezeigt, dass die Themen der Berliner Deklaration von 2007 unverändert aktuell sind und weitere, nachhaltige Anstrengungen erforderlich sind, um HIV/TB als „double trouble“ zu einem „double success“ werden zu lassen. Einiges ist bisher erreicht worden, und es gibt noch viel zu tun! ${ }^{7}$

\section{Interessenkonflikte}

$\nabla$

Die Autoren geben an, dass kein Interessenkonflikt besteht.

\section{Literatur}

1 World Health Organization. Plan to stop TB in 18 high priority countries in the WHO European Region, 2007-2015. Genf: WHO, 2007

2 European Centre for Disease Prevention and Control (ECDC). Framework action plan to fight tuberculosis in the European Union. Stockholm Schweden: Februar 2008

3 World Health Organization. The Stop TB Strategy. Building on and enhancing DOTS to meet the TB-related Millenium Development Goals. WHO/HTM/STB/2008. Genf: World Health Organization, 2006

4 Europäisches Ministerforum der Weltgesundheitsorganisation (WHO). „All against tuberculosis“ am 22. Oktober 2007 in Berlin.www.euro. who.int/tuberculosis/TBForum/2007

\footnotetext{
7 Wir danken dem deutschen Bundesministerium für Gesundheit für die Unterstützung.
} 
5 World Health Organization. Global tuberculosis control: epidemiology, strategy, financing. WHO/HTM/TB/2009.411. Geneva, Switzerland: WHO,

6 World Health Organization. Anti-tuberculosis drug resistance in the world. Report No. 4, 2008. WHO/HTM/TB/2008.

7 The Work of WHO in the Eastern Mediterranean Region. Annual Report of the Regional Director. 1 January-31 December 1995. www. emro.who.int/stb/media/pdf/RDReport_95.pdf

8 Podlekareva DN et al. Mortality from HIV and TB coinfections is higher in eastern Europe than in Western Europe and Argentina. AIDS 2009; 23: $2485-2495$

9 Annual epidemiological report on communicable diseases in Europe 2009. Surveillance report. Stockholm/Schweden: European Centre for Disease Prevention and Control, 2009

10 Gamazina $K$ et al. Improving quality of prevention of mother-to-child HIV transmission services in Ukraine: a focus on provider communication skills and linkages to community-based non-governmental organizations. Cent Eur J Public Health 2009; 17: 20 - 24

11 UNAIDS/WHO. Epidemiological Fact Sheets on HIV and AIDS. Core data on epidemiology and response. Update. Ukraine: 2008

12 Dubrovina I et al. Drug-resistant tuberculosis and HIV in Ukraine: a threatening convergence of two epidemics? Int J Tuberc Lung Dis 2008; 8: $756-762$

13 Mosneaga A, Yurasova E, Zaleskis R, Jakubowiak W. Enabling health systems in tuberculosis control: challenges and opportunities for the former Soviet Union countries. In: European Observatory for Health Systems and Policies Series. Coker R, Atun R, McKee M, eds. Health Systems and the Challenge of Communicable Diseases: Experience from Europe and Latin America. Maidenhead, England: McGraw-Hill, Open University press, 2008: $171-192$

14 Ottenhoff TH. Overcoming the global crisis: „yes, we can“, but also for TB...? Eur J Immunol 2009; 39: 2014-2020 